\title{
Cardiovascular Risk in Diabetes Mellitus: Cause and Effect
}

\author{
Charles V. Pollack Jr. • William T. Ford • \\ Gregory Volturo $\cdot$ Sameer Badlani $\cdot$ \\ David J. Yu • Gerard X. Brogan Jr.
}

Published online: 31 December 2013

(c) Springer Science+Business Media New York 2013

\begin{abstract}
In the care of the diabetic patient being admitted to the hospital from the emergency department (ED), there is an opportunity to provide a smoother transition of care by recognizing that the first $6-12 \mathrm{~h}$ of care have an important impact on the outcomes and overall length of hospital stay. This is especially true when an inpatient bed is not immediately available for transfer and the burden of initial care falls on the emergency physician and nurses. Although it varies across the country, for many patients, the first 12 or more hours of "inpatient care" may be spent in the ED, representing perhaps as much as onethird of the entire hospital stay. With the rapid growth and influence of Accountable Care Organizations and focus on value over volume, length of stay is more critical than ever. It is more important to initiate the correct dose of insulin, along with a thorough evaluation of the diabetic patient's other medications with the aim to stabilize the patient for a more effective hand-off to the hospitalist, cardiologist, surgeon, or any other inpatient provider. The value of this type of coordinated care is enhanced in patients who also have cardiovascular disease (CVD) because of the benefits associated with optimal glycemic control. In this article, we will review the current definition of optimal glycemic control as it applies to the patient with diabetes who also has cardiovascular disease, in order to highlight procedural changes that can be made to facilitate overall quality care. Underlying this call for a new focus is the observation that the impact of tighter glycemic control on outcomes in CVD
\end{abstract}

C. V. Pollack Jr. $(\bowtie)$ · W. T. Ford · G. Volturo · S. Badlani · D. J. Yu - G. X. Brogan Jr.

Department of Emergency Medicine, Pennsylvania Hospital, University of Pennsylvania, Philadelphia, PA, USA

e-mail: cvpollack@gmail.com such as acute coronary syndrome, heart failure, sepsis, and stroke cannot be underestimated.

Keywords Cause of cardiovascular risk in diabetes mellitus - Effect of cardiovascular risk in diabetes mellitus . Cardiovascular risk in diabetes mellitus - Cardiovascular risks - Diabetes - Diabetes mellitus - Heart conditions and diabetes

\section{Introduction}

The transition of care from the emergency department (ED) to the hospital medicine service represents a common step in the continuum of care but its impact may be underappreciated, especially in patients who present with diabetes mellitus (DM) and may have significant comorbidities. Blood glucose levels may be particularly labile in an ill patient with diabetes while in the ED and on the hospitalist's service. Optimal transitions of care are more likely when emergency physicians (EPs) and receiving inpatient providers are proactively attentive to metabolic factors.

This article and the two that follow reflect the collaborative work of EPs and hospitalists within the Hospital Quality Foundation (HQF), a nonprofit professional organization dedicated to improving the practice of hospital medicine. As part of these efforts, HQF has developed the "HEMI" (Hospital-Emergency Medicine Interface) educational initiative to bring into sharp focus interventions and programs aimed at supporting the evidence basis and improving consistent delivery of "best practice" care for patients managed across the transition from ED to hospital medicine, and, ultimately, back out to the community.

Emergency physicians and hospitalists are responsible for much of the early assessment and management of 
patients with diabetes and its comorbidities and complications. Their decisions have broad short- and long-term ramifications and ideally should be based on similar interpretations of the evidence that are consistently applied.

To discuss the concept of optimizing collaborative care of the diabetic patient in the upstream environment, an expert panel of EPs and hospitalists met in Philadelphia, PA, USA, August 1-2, 2013, to discuss their perspectives on the evidence around the continuum of care for patients who present to the ED with diabetes and require hospitalization. The meeting was supported by an unrestricted educational grant from Sanofi Pharmaceuticals. In this report, the HEMI-DM panel examined the impact of diabetes on acute cardiovascular presentations.

\section{Diabetes Mellitus}

The clinical significance between DM and comorbid conditions is highlighted in the relationship between ACS and diabetes [1••]. The incidence of diabetes among ACS patients ranges from 15 to $35 \%$. It is also important to consider the number of ACS patients who may have undiagnosed diabetes and could, therefore, benefit from a broader approach to their ACS treatment regimen. Additionally, a subset of patients with diabetes have unrecognized renal insufficiency, a factor that may impact proper drug dosing and length of hospital stay. Finally, approximately $35 \%$ of the US population is prediabetic [1 ••], which may further impact their overall CVD risk profile.

The concern about diabetes is not just related to acute care in the ED. Diabetes is a chronic disease with potentially serious consequences. Over time, diabetes results in damage and dysfunction in multiple organ systems. Vascular disease is a major cause of many of the sequelae of long-term disordered glucose metabolism. Both microvascular disease (retinopathy, nephropathy, and neuropathy), that is specific to diabetes, and macrovascular disease (involving the coronary, cerebral, and peripheral vascular supplies), that occurs with increased frequency in diabetes, contribute to the high morbidity and mortality rates associated with this disease [2•].

Atherosclerotic macrovascular disease occurs with increased frequency in diabetes, resulting in an increased incidence of myocardial infarction, stroke, claudication and gangrene of the lower extremities. Although macrovascular disease accounts for significant morbidity and mortality in both type 1 (T1D) and type 2 diabetes (T2D), the effects of large-vessel disease are particularly devastating in Type 2 DM and are responsible for approximately $75 \%$ of diabetes-related deaths $[2 \cdot, 3]$.

Diabetes is traditionally classified as type 1 or type 2 . In the past, type 1 has also been termed "juvenile-onset" and "insulin-dependent," and type 2 was referred to as "adultonset" and "non-insulin-dependent" diabetes. As the pathophysiology of the disease has been better elaborated, treatment approaches have evolved, and the incidence of childhood obesity has increased, these distinctions are now not so clear. A better way to differentiate the two is by considering the amount and effectiveness of basal insulin in the individual patient's system; patients who have a true insulin deficiency are type 1 , and require insulin replacement therapy. Those who make insulin but either in insufficient amounts (as in many morbidly obese diabetic patients) or in the presence of reduced cellular insulin receptor activity are designated type 2 and may be treated with oral agents, supplemental insulin, behavioral and dietary changes, or all of these [2•].

Diabetes increases the risk of atherosclerosis in at least three ways: (1) the incidence of traditional risk factors, such as hypertension and hyperlipidemia, is increased among diabetics (50 and $30 \%$ incidence at diagnosis, respectively); (2) diabetes itself is an independent risk factor for atherosclerosis; and (3) diabetes appears to synergize with other known exogenous risk factors (such as smoking and obesity) to increase atherosclerosis. The elimination of other risk factors, therefore, can greatly reduce the risk of atherosclerosis in diabetes [2•,3].

To better understand the clinical implications of this, it will be useful to take a closer look at the pathophysiological relationship between elevated glucose levels and vascular disease.

\section{Pathophysiology}

Vasculitis is an important component of the diabetes syndrome. Components of the immune system are altered in obesity and T2D, with the most apparent changes occurring in adipose tissue, the liver, pancreatic islets, the vasculature, and circulating leukocytes. Inflammatory mechanisms in T2D may include local hypoxia, premature death of lipid cells, and metabolic stresses associated with insulin resistance. Metabolic stresses result in subsequent activation of stress-induced proinflammatory cytokines, which may be associated with the recruitment of inflammatory cells [4]. Circulating inflammatory cells and immune cells constitute an important part of an atheroma [5], and diabetes accelerates the process of atherosclerosis and, therefore, predisposes the patient to CVD.

Data indicate that high intracellular levels of glucose in cells that cannot down-regulate glucose entry result in microvascular damage via four distinct, diabetes-specific pathways: (1) increased polyol pathway flux, (2) increased formation of advanced glycation end-product, (3) activation of protein kinase C, and (4) increased hexosamine pathway flux. 
More recent information suggests that increased flux through these four pathways is induced by a common factor: mitochondrial-derived reactive oxygen species, which are generated by increased flux of glucose through the tricarboxylic cycle. The end result of these changes in the microvasculature is an increase in protein accumulation in vessel walls, a loss of endothelial cells, and, ultimately, occlusion. Evidence suggests that all four of these pathways may actually be linked by a common mechanistic element: hyperglycemia-induced oxidative stress.

The common pathway between DM and coronary artery disease may be endothelial dysfunction. Lumsden and colleagues conducted a single visit study to examine whether endothelial function was normal following aggressive treatment of cardiovascular risk factors in 15 patients with T2D and a history of ACS [6]. At the end of the study, total and low-density lipoprotein cholesterol levels were well controlled. However, other risk factors, such as increased body mass index, increased systolic blood pressure, glucose, and triglyceride levels, and lower high-density lipoprotein levels were still present. Analysis of endothelial function using several different methods revealed that these patients with T2D exhibit residual cardiovascular risk factors despite being on standard clinical care. They continue to show evidence of endothelial dysfunction, which has been shown to be an independent predictor of cardiovascular events. Thus, providing optimal glycemic control may provide an additional benefit to patients with CVD through its impact on endothelial function [6].

With the inextricable relationship between DM and CVD in mind, it is clear that identification of diabetes in patients who also have CVD is essential for a smooth transition of care to the inpatient service. The treatment process can begin in the ED after the patient's condition is known and can continue in an uninterrupted fashion by the hospitalist or cardiologist who manages these patients in the hospital. The initiation of aggressive secondary prevention measures while the patient is in the ED may not be common practice today; however, there appears to be an opportunity to start the patient on the proper courseperhaps at a "teachable moment" in the ED—as he or she is actually admitted to the hospital.

\section{Incidence and Epidemiology}

DM is an important consideration for both the emergency physician and the hospitalist because of the growing number of patients with this disease who become part of the ED and hospital population. The 2011 National Diabetes Fact Sheet states that approximately 25.8 million people have DM in the United States, which represents over $8 \%$ of the population. Of those, 18.8 million carry a diagnosis but seven million remain undiagnosed. An additional 79 million people ${ }^{1}$ have prediabetes. There were approximately 1.9 million new cases of diabetes diagnosed in people aged 20 years and older in 2010. The disease is relatively more common in African-Americans and Hispanics [7]. If diabetes was an isolated disease, these striking epidemiological figures would not seem as important. However, because DM is often associated with one or more comorbidities, DM is becoming one of the key factors driving hospitalization.

In 2007, diabetes was listed as the underlying cause on over 70,000 death certificates and was listed as a contributing factor on more than 160,000 death certificates: over 230,000 deaths in 1 year, and the number is growing. In addition to mortality risk, diabetes is associated with target organ damage in several different systems [7]. For example, in 2004, heart disease was noted on almost $70 \%$ of diabetes-related death certificates among people aged 65 years or older and stroke was noted on $16 \%$ of death certificates for the same group. It is generally accepted that adults with diabetes have heart disease death rates about two to four times higher than adults without diabetes and at least a similar risk for stroke [7].

Diabetes is the leading cause of kidney failure, accounting for over $40 \%$ of new cases in 2008. In that same year, over 48,000 people with diabetes began treatment for end-stage kidney disease in the United States and more than 202,000 with end-stage kidney disease due to diabetes were on chronic dialysis or had received a kidney transplant [7].

Diabetic patients have a higher incidence of kidney disease because of microvascular damage to the glomerulus and small renal vessels. Baber and Auguste pointed out that chronic kidney disease and DM are highly prevalent among patients presenting with acute coronary syndromes. Consistent with the arguments already presented, they suggested that residual vascular risk remains disproportionately high in these populations primarily due to preexisting vascular morbidity and the increase in overall cardiovascular risk occurs as a result [8•].

They further hypothesized that the interaction between elements of circulating blood and the atherosclerotic plaque, culminating in thrombotic events such as myocardial infarction, may be even more important in patients with chronic kidney disease and DM. Both diseases are prothrombotic, inflammatory conditions resulting in

\footnotetext{
${ }^{1}$ In contrast to the 2007 National Diabetes Fact Sheet, which used fasting glucose data to estimate undiagnosed diabetes and prediabetes, the 2011 National Diabetes Fact Sheet uses both fasting glucose and A1C levels to derive estimates for undiagnosed diabetes and prediabetes. These tests were chosen because they are most frequently used in clinical practice.
} 
atherothrombosis. In DM, pathologic abnormalities such as insulin resistance and hyperglycemia directly modulate platelet function and may lead to high levels of platelet reactivity. In patients with chronic kidney disease, high levels of circulating procoagulant factors and abnormalities of nitric oxide synthesis may account for platelet hyperreactivity. Because of these additional factors, they proposed that the introduction of novel antiplatelet and antithrombotic agents may provide an opportunity to improve the adverse prognosis among patients with ACS who also have chronic kidney disease and DM [8•]. Please see Table 1 for diagnostic criteria for the diabetes.

\section{Diabetes Mellitus as a Potent Metabolic Factor}

\section{Prediabetes}

The term "prediabetes" was initially used to identify patients with borderline glucose levels under physiologically stressful conditions, such as pregnancy, or in overweight persons with a strong family history of T2D. Because many of these individuals never actually developed clinical diabetes, another definition was established by the World Health Organization [10] and later refined by the American Diabetes Association. The current definition is based on impaired glucose tolerance (IGT), impaired fasting glucose (IFG), and hemoglobin A1c (HbA1c) of 5.7-6.4\%, but not on the presence or absence of other risk factors for diabetes, [2•] although this definition is not universally accepted.

An important reason to identify patients with prediabetes is its relationship to the metabolic syndrome. An elevated glucose level is one component of the current

Table 1 Diagnostic criteria for the diabetes $[1 \bullet \bullet]$

Diagnostic criteria
The most current American Diabetes Association criteria for the
diagnosis of diabetes includes the following:
1. HbA1C $>6.5 \%$ a b; or
2. Fasting plasma glucose $(\mathrm{FPG})^{\mathrm{c}}>126 \mathrm{mg} / \mathrm{dL}(7.0 \mathrm{mmol} / \mathrm{L})^{\mathrm{b}}$; or
3. 2-h plasma glucose $\geq 200 \mathrm{mg} / \mathrm{dL}(11.1 \mathrm{mmol} / \mathrm{L})$ during an oral
glucose tolerance test $(\mathrm{OGTT})(75 \mathrm{~g})$; or
4. Random plasma glucose $\geq 200 \mathrm{mg} / \mathrm{dL}(11.1 \mathrm{mmol} / \mathrm{L})^{\mathrm{d}}$
Note that the above recommendations are for nonpregnant adults
a Perform in lab using a NGSP-certified method standardized to the
DCCT assay
b ln the absence of unequivocal hyperglycemia result to be confirmed
by repeat testing
c Fasting defined as no caloric intake for $\geq 8 \mathrm{~h}$
d $\ln$ persons with symptoms of hyperglycemia or hyperglycemic
crisis

consensus definition of the metabolic syndrome, along with abdominal obesity - a key factor for increased cardiovascular risk-elevated blood pressure, elevated triglycerides, and reduced high-density lipoprotein (HDL) cholesterol. Any three of these five components confer a diagnosis of the syndrome [9]. Excess adipose tissue releases abnormally high levels of fatty acids and a variety of other factors that predispose to both diabetes and CVD.

The occurrence of microvascular pathology, usually manifest as retinopathy, glomerular disease, or neuropathy, has been used to differentiate between prediabetes and diabetes. Hyperglycemia appears to target endothelial cells, causing thickening of the membrane beneath endothelial cells as a fundamental structural change [9]. There is no specific plasma glucose threshold for the development of microangiopathy [10].

An unresolved question is whether elevated glucose levels are a direct cause of atherosclerosis or clinical CVD. A systematic review of the relationship between prediabetes and CVD risk suggests that prediabetes imparts a modest increase in risk for CVD, such as in the range of $20 \%$. Identification of these patients in the ED or hospital can initiate a more comprehensive review of their metabolic status and considerations for how these patients should be followed.

\section{Diabetes}

There is a clear relationship between overt diabetes and cardiovascular risk. Giraldez and colleagues reported the results of an analysis of the data from the EARLY ACS trial in which patients who presented with non-ST-segment-elevation (NSTE) ACS and for whom an invasive strategy was planned, were evaluated for signs of diabetes $[1 \bullet \bullet]$. A known diagnosis of diabetes was recorded on the case report form, as were hemoglobin A1c (HbAlc) and fasting blood glucose values for all patients. Patients were stratified into four categories (Table 2).

The primary efficacy outcome of the study was the 30-day composite of all-cause death or myocardial infarction (MI), but, in addition, the relationship between diabetes grouping and 30-day mortality, 30-day death, MI, or

Table 2 Patient stratification

\begin{tabular}{ll}
\hline Category & Description \\
\hline Known diabetes & $\begin{array}{c}\text { Either reported on the CRF or use of glucose- } \\
\text { lowering drugs at admission }\end{array}$ \\
$\begin{array}{l}\text { Undiagnosed } \\
\text { diabetes }\end{array}$ & $\begin{array}{c}\text { Fasting glucose } \geq 126 \mathrm{mg} / \mathrm{dL} \text { or HbA1c } \geq 6.5 \% \\
\text { with no previous diabetes history }\end{array}$ \\
$\begin{array}{l}\text { Prediabetes } \\
\text { Normal }\end{array}$ & Fasting glucose $110-126 \mathrm{mg} / \mathrm{dL}$ \\
\hline
\end{tabular}


recurrent ischemia requiring urgent revascularization (RIUR), and 1-year all-cause mortality was evaluated.

The primary endpoint occurred in $13.8 \%$ of patients in the undiagnosed diabetes group, compared with $10.7 \%$ of non-diabetic patients. Thirty-day death or MI occurred in $12.1 \%$ of prediabetic patients and in $12.8 \%$ of diabetic patients, but these rates were not significantly different from normal patients (Fig. 1).

The endpoint of 30-day death, MI, or RIUR was more frequent, however, in both the undiagnosed and known diabetes groups compared with normal patients. All-cause mortality at 30 days among patients with known diabetes and undiagnosed diabetes was more frequent than among normal patients, but similar among prediabetic and normal patients. Survival analyses confirmed that 1-year mortality among patients with known diabetes was significantly greater than among normal patients, even after adjustments for confounding factors $[1 \bullet \bullet]$.

The investigators concluded that a substantial proportion of patients admitted with high-risk non-ST-segment ACS had previously undiagnosed DM $(12.2 \%)$ or prediabetes (10.8\%), as defined by fasting glucose or HbA1c after hospital admission. Compared with patients without diabetes, patients with undiagnosed diabetes had poorer early outcomes, characterized by increased rates of all-cause death or MI and all-cause death alone at 30 days. Similarly, compared with normal patients, those with known diabetes had a significantly greater 30 -day mortality risk [1••]. Early recognition of this risk could drive more aggressive therapy in both the ED and the hospital.

\section{Effects of Glycemic Control on Cardiovascular Risk}

The focus on identifying patients with clinical diabetes or prediabetes who present to the ED with CVD is not only based on the observation that these patients tend to have

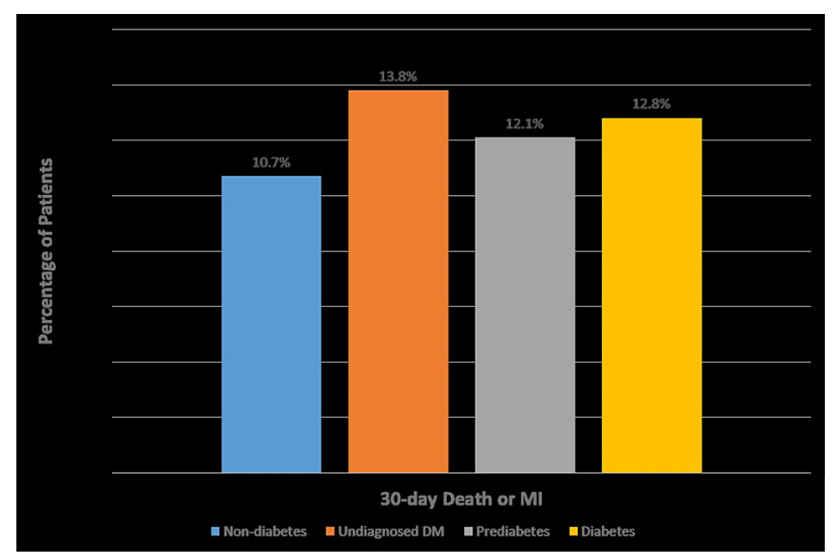

Fig. 1 Percentage of patients reaching primary endpoint $[1 \bullet \bullet]$ poorer outcomes if they have diabetes. It is also the result of a more complex relationship between hyperglycemia, cardiovascular risk, and a third factor, renal insufficiency. DM is a known risk factor for CVD and plays a role in worsening the patient's prognosis when in the presence of coronary artery disease, heart failure, hyperlipidemia and peripheral artery disease.

\section{Impact on Coronary Artery Disease}

Patients with DM have a threefold risk for acute coronary syndrome [11, 12, 13•, 14, 15] and patients with diabetes have coronary events 15 years sooner than those without diabetes $[11,12,13 \bullet, 14]$. This negative relationship also extends to heart failure as well.

Evidence supporting the importance of early detection of DM in patients who present with ACS was provided by Niccoli and colleagues, who evaluated two different patient populations with a first ACS and were enrolled for angiographic and optical coherence tomography studies. Angiographic CAD severity was assessed, as well as the development of collateral vessels. In the angiographic components of the study, diabetes was positively associated with both the stenosis score and the extent of disease. Interestingly, well-developed collateral circulation towards the occluded or partially-occluded vessel was more frequent in diabetic than in non-diabetic patients. The lipid arc appeared to be smaller in diabetics than in non-diabetic patients, and the most calcified cross-section along the diseased segment of coronary artery had a greater number of calcified quadrants and a wider calcified arc in diabetic than in non-diabetic patients. The authors concluded that diabetic patients exhibit substantially more severe coronary atherosclerosis than non-diabetic patients at the time of a first acute coronary event, along with better collateral development towards the primary diseased vessel. The potent pro-inflammatory, pro-oxidant and pro-thrombotic stimuli operating in T2D may play a role [16].

\section{Heart Failure}

Diabetes has also been identified as a risk for HF that is partly but not entirely linked to coronary heart disease and hypertension. For example, associations have also been reported between absolute blood glucose levels, success of glycemic control, and HF. The Framingham Study firmly established the epidemiologic link between diabetes and HF [17]. The risk of HF was increased 2.4-fold in diabetic men and fivefold in diabetic women. When patients with prior coronary or rheumatic heart disease were excluded, the relative risk of $\mathrm{HF}$ remained elevated at 3.8 in diabetic men and 5.5 in diabetic women. 
Similar findings have since been noted in a number of other studies. In a report of 9,591 subjects with T2D and matched controls, HF was more frequent at baseline in diabetics (11.8 vs $4.5 \%)$. Among those free of HF at baseline, HF developed more often in diabetics during a 30-month follow-up. The incidence of HF in diabetic patients is even higher among elderly adults, who comprise the fast-growing demographic of ED patients [18].

A report from Kaiser Permanente of over 48,000 adults (mean age 58 years) with DM (predominantly T2D) and no evidence of $\mathrm{HF}$ were followed for a mean of 2.2 years. After adjustment for covariates, each $1 \%$ increase in $\mathrm{HbA1c}$ was associated with an $8 \%$ increased relative risk of HF [19].

A similar relationship was found between blood glucose levels and hospitalization for HF in a cohort of 31,546 adults (mean age 67 years) with high cardiovascular risk [20]. At a mean follow-up of 2.4 years, each $18 \mathrm{mg} / \mathrm{dL}$ $(1 \mathrm{mmol} / \mathrm{L})$ increase in baseline fasting plasma glucose was associated with a modest but statistically significant increase in the risk of hospitalization for heart failure.

Although the exact causal relationship has not been conclusively identified, small vessel disease, autonomic neuropathy, diastolic stiffness and decreased insulin availability to cardiac muscle have been proposed as a possible link between DM and HF.

\section{Hyperlipidemia}

DM may also have an impact on the severity of hyperlipidemia. The typical lipoprotein pattern in DM consists of moderate elevation in triglyceride levels, low HDL cholesterol values, and small dense LDL particles. This lipoprotein pattern is associated with insulin resistance and is present even before the onset of diabetes. Small dense LDL particles are highly atherogenic because of their enhanced susceptibility to oxidative modification and increased uptake by the arterial wall. At triglyceride levels $>132 \mathrm{mg} /$ $\mathrm{dl}$, small LDL particles become common. It is well known that LDL cholesterol is one of the strongest independent predictors of CAD followed by low HDL cholesterol. Because hyperlipidemia and elevated blood glucose are both part of the metabolic syndrome, the natural question is whether there is a causal relationship between DM and hyperlipidemia as well—or at least a common pathophysiological pathway.

Al-Aqeedi and colleagues conducted a prospective study of 467 consecutive male patients hospitalized for ACS [21]. Of the 467 patients, $324(69.4 \%)$ fulfilled the criteria for metabolic syndrome. The most common abnormal metabolic components were elevated fasting blood glucose, reduced high-density lipoprotein cholesterol, and elevated triglycerides. The authors concluded that in ACS patients without previous history of DM, metabolic syndrome is highly prevalent and that elevated fasting blood glucose levels are an important part of that clinical spectrum. Whether the elevated glucose levels contributed to hyperlipidemia in these patients is unclear; however, elevated glucose levels clearly represent an important part of the relationship between metabolic syndrome and ACS.

\section{Peripheral Artery Disease}

Diabetes is also a risk factor for peripheral artery disease (PAD), which most commonly affects the arteries supplying the lower extremities. Restriction of blood flow due to arterial stenosis or occlusion often leads to intermittent claudication. Any further reduction in blood flow causes ischemic pain at rest, which can even affect the foot. Ulceration and gangrene may then supervene and can result in loss of the limb if not treated [22].

PAD affects approximately 12 million people in the US [23]. Data from the Framingham Heart Study revealed that $20 \%$ of symptomatic patients with PAD had diabetes, but this probably underestimates the prevalence, since more people with PAD are asymptomatic than symptomatic [24]. Diabetes and smoking are the strongest risk factors for PAD [23].

Inflammation was already discussed as a key pathophysiological component in the development of vascular disease. It has also been established as both a risk marker and perhaps a risk factor for PAD. Elevated levels of C-reactive protein (CRP) are strongly associated with the development of PAD. Interestingly, levels of CRP are abnormally elevated in patients with impaired glucose tolerance and diabetes and may be involved with changes in the endothelium of major vessels. The endothelial lining of the arterial vasculature modulates the relationship between the cellular elements of the blood and the vascular wall, mediating the normal balance between thrombosis and fibrinolysis. The endothelial lining also plays an integral role in interactions between leukocytes and the cell wall. Thus, elevated blood glucose levels, perhaps through a common factor of CRP, may contribute to the inflammatory process and endothelial dysfunction associated with atherosclerosis and adverse outcomes [23]. In addition to PAD in the legs, another vascular system that appears to be affected by diabetes and hyperglycemia is the cerebral circulation.

\section{Stroke}

Diabetes appears to play a role in the risk for cerebrovascular accident. In addition, incident hyperglycemia has 
been reported to augment acute ischemic brain injury in many human and animal studies. Possible mechanisms include increased brain tissue acidosis, increased bloodbrain barrier permeability, and increased hemorrhagic transformation of the infarct. Whether acute hyperglycemia independently affects patient outcomes or whether this effect primarily reflects the effects of increased stroke severity or poor glycemic control has not been established [25].

\section{Renal Insufficiency}

Some patients who present to the ED may also have undiagnosed renal insufficiency. Kim and associates examined the effect of metabolic syndrome on clinical outcomes in patients with acute myocardial infarction (AMI) in the presence or absence of renal insufficiency. They enrolled 11,462 patients with AMI in a prospective Korean Acute Myocardial Infarction Registry. Participants were analyzed according to the presence or absence of both metabolic syndrome and renal insufficiency, with the primary endpoint being major adverse cardiac events, which included a composite of all-cause death, myocardial infarction, target lesion revascularization, and coronary artery bypass graft during the 1-year follow-up period. The results revealed that metabolic syndrome had a higher prevalence in patients with AMI who also had evidence of renal insufficiency with low glomerular filtration rate. Inhospital death and the composite endpoint were significantly higher in patients with metabolic syndrome than in those without. The 1-year mortality rate was also higher in patients with both metabolic syndrome and renal insufficiency, suggesting that the presence of renal insufficiency may have a negative impact on outcomes [26]. Diabetes is one of several causes of renal insufficiency, which may not always be clinically apparent when the patient presents.

Although the clinical implications of these findings is not completely clear, the authors suggested that CVD follows a different course in patients with DM with or without renal insufficiency but that renal insufficiency, if present, may play an additive role. Thus, patients with diabetes or prediabetes may benefit from being identified in the ED to allow for an alternate management strategy, which may include dose adjustments for diminished renal function or the choice of different agents.

\section{Optimal Management of Patients with Diabetes}

When patients with prediabetes or DM come to the hospital, there is an opportunity to create a positive treatment continuum that begins in the ED and transitions to the inpatient service. This can be facilitated by the development of standard protocols to detect DM when the patient enters the ED and an ongoing collaborative relationship between the EPs and the hospitalist or other hospital-based medical personnel. With these systems in place, the goal would be to provide optimal glycemic control for the patient from the time he or she arrives until discharge. However, optimal glycemic control does not necessarily mean that the patient's blood glucose level is maintained between 80 and $100 \mathrm{mg} / \mathrm{dL}$ at all times, nor does it mean that the same approach should be used in every patient. Insulin therapy is the recommend inpatient strategy and oral hyperglycemic medication should be discontinued until the patient is ready for discharge. Optimal glycemic control in a hospital setting will most likely be successful if the patient is managed with insulin therapy, so that accommodations can be made for changes that occur as a result of the underlying illness, therapeutic interventions or other factors that may not exist in the patient's home environment. Insulin therapy can begin in the ED and preferably with basal insulin.

\section{The Importance of Glycemic Control}

Optimal glycemic control is important for all critically ill patients [27-29]. In both the ED and hospital settings, insulin therapy is the preferred method for achieving glycemic control in most clinical situations. When possible, IV infusion is the preferred route of insulin administration; however, outside of acute treatment areas and critical care units, subcutaneous administration of insulin is used much more frequently. Orally administered agents should have a limited role in the inpatient setting [30••].

Continuous IV insulin infusion has been shown to be the most effective method for achieving specific glycemic targets in patients treated in the intensive care unit. Because of the very short half-life of circulating insulin, continuous IV delivery allows rapid dosing adjustments to address alterations in the status of patients. It is ideally administered by means of validated protocols that allow for predefined adjustments in the insulin infusion rate based on glycemic fluctuations and insulin dose [2•].

In a meta-analysis of the use of insulin therapy for critically ill hospitalized patients, Pittas and colleagues analyzed 35 trials. Combining data from all trials demonstrated that insulin therapy decreased short-term mortality by $15 \%$. In a subgroup analysis, insulin therapy decreased mortality in the surgical intensive care unit, when the aim of therapy was glucose control in patients with DM. A near-significant trend toward decreasing mortality was seen in patients with acute myocardial infarction who did not receive reperfusion therapy. The authors concluded that 
insulin therapy initiated in the hospital in critically ill patients has a beneficial effect on short-term mortality in different clinical settings [31].

Optimal glycemic control can have a positive effect on patient morbidity, length of hospital stay and the overall cost of care. In the DIGAMI 1 study, (Diabetes Mellitus Insulin-Glucose Infusion in Acute Myocardial Infarction) intensive insulin therapy for diabetic patients who were experiencing an acute myocardial infarction included an insulin-glucose infusion during the initial $24 \mathrm{~h}$ of hospitalization, followed by subcutaneous insulin four times daily for a minimum of 3 months [32]. Morbidity and mortality were assessed in the acute, subacute, and chronic phases. Acute effects, determined during the first 12-24 h after MI, included assessment of initial infarct size. Subacute effects ( $2-30$ days) and chronic effects ( $>30$ days) included the incidence of significant arrhythmias, reinfarction, congestive heart failure, and death.

The insulin-treated and the control groups had an approximately equal number of in-hospital complications, including ventricular fibrillation, re-infarction, high degree atrioventricular conduction disturbances, and congestive heart failure. Regardless of the treatment protocol received, the in-hospital courses were similar. Overall, patients had a mortality of $10.2 \%$ in the hospital, $14.0 \%$ at 3 months, and $22.4 \%$ at 1 year. Though the infusion group had a slightly lower mortality than the control group in the hospital, at 3 months post-MI, and at 1 year, only the 1-year mortality was statistically significant. When examining mortality in the pre-stratified risk groups, the greatest mortality reduction is noted for patients who had never been on insulin before and were classified as low cardiac risk [32].

The DIGAMI 2 study was an international trial involving 3,000 subjects with a trial design similar to that of DIGAMI 1, enrolling persons with known diabetes or with initial glucose $>11 \mathrm{mM} / \mathrm{L}(198 \mathrm{mg} / \mathrm{dL})$ presenting with AMI with duration of symptoms $<24 \mathrm{~h}$. Study participants were randomized to 1 of 3 groups. Patients in the first group had a glucose/insulin infusion for at least $24 \mathrm{~h}$ and then were treated with subcutaneous insulin. The second intervention group was treated acutely with glucose/insulin infusion for at least $24 \mathrm{~h}$ and then returned to conventional treatment. The third intervention group received conventional treatment only. Although the study was discontinued early due to low recruitment, DIGAMI 2 did not support the acute introduction of long-term insulin treatment to improve survival in type 2 diabetic patients following myocardial infarction, when compared with a conventional management at similar levels of glucose control. In addition, insulin-based treatment did not lower the incidence of non-fatal myocardial reinfarction and stroke. However, an epidemiological analysis confirmed that the glucose level was a strong, independent predictor of long-term mortality in this patient category, underlining that glucose control seems to be an important part of their management [33].

Optimal glycemic control is also an important issue for patients with stroke. Williams et al. studied patients hospitalized with acute ischemic stroke over a 5-year period. Admission stroke severity, serial blood glucose levels, and new diabetes diagnoses were recorded. Hyperglycemia at admission to hospital was present in $40 \%$ of patients with acute stroke. Patients with hyperglycemia were more often women and more likely to have prior diagnoses of diabetes and heart failure. In stroke patients, hyperglycemia independently increased the risk for death at 30 days, 1 year, and 6 years after stroke. The authors concluded that hyperglycemia at the time of admission was common among patients with acute ischemic stroke and was associated with increased short- and long-term mortality. They also suggested that a trial of intensive treatment of hyperglycemia should be considered for patients with acute stroke [25].

In another study, Alverez-Sabin and colleagues evaluated 268 consecutive patients with a nonlacunar middle cerebral artery (MCA) stroke at $<3 \mathrm{~h}$ after onset, $27.2 \%$ of whom were treated with tissue plasminogen activator (tPA) [34]. Serum glucose was determined at baseline before tPA administration. The results revealed that at baseline, 31 patients $(42.5 \%)$ were hyperglycemic. Early reperfusion $(<6 \mathrm{~h})$ occurred in 43 patients $(58.9 \%)$; however, admission blood glucose correlated negatively with the degree of neurological improvement at $24 \mathrm{~h}$ in reperfused but not in nonreperfused tPA-treated patients. Factors associated with poor outcome were increased age, history of DM, admission glucose $>140 \mathrm{mg} / \mathrm{dL}$, and early reocclusion among reperfused patients. Only admission glucose value $>140 \mathrm{mg} / \mathrm{dL}$ emerged as an independent predictor of poor outcome despite tPA-induced recanalization. The authors concluded that hyperglycemia before reperfusion may in part counterbalance the beneficial effect of early restoration of blood flow, which translates into a worse outcome in hyperglycemic patients despite tPA-induced recanalization.

\section{Implications for the ED and Hospital Services}

DM is frequently not diagnosed until complications appear, and approximately one-quarter of all people with diabetes in the US may be undiagnosed [2•]. The effectiveness of early identification of prediabetes and diabetes through mass testing of asymptomatic individuals has not been validated, and rigorous trials to provide such proof are unlikely to occur.

Recognition of the disease in the ED and inpatient setting is, however, useful. Testing to detect T2D and 
prediabetes in asymptomatic people should be considered in adults of any age who are overweight or obese (BMI $\geq 25 \mathrm{~kg} / \mathrm{m}^{2}$ ) and who have one or more additional risk factors for diabetes. ${ }^{2}$ In those without these risk factors, testing should begin at age 45 years. If tests are normal, repeat testing at least at 3-year intervals is reasonable. To test for diabetes or prediabetes, the A1C, FPG, or 75-g 2-h OGTT are appropriate tests. In those identified with prediabetes, identify and, if appropriate, treat other CVD risk factors such as hyperlipidemia, elevated blood pressure and obesity.

Patients with diabetes are more likely to be hospitalized and to have longer durations of hospital stay than those without diabetes. From the standpoint of treatment, as mentioned previously, the 2009 ADA/AACE Guidelines suggest that patients with diabetes should be switched from oral agents to insulin therapy while they are inpatients [30••]. The management of hyperglycemia in the hospital presents unique challenges that stem from variations in a patient's nutritional status and level of consciousness, dietary restrictions for diagnostic testing, the practical limitations of intermittent glycemic monitoring, and the ultimate importance of patient safety. Accordingly, reasonable glucose targets in the hospital setting are modestly higher than may be routinely advised for patients with diabetes in the outpatient setting, and adequate control may best be achieved by switching a patient from oral agents to insulin [30••].

Together, these studies suggest that identification of patients with diabetes before they are admitted to the hospital may provide an opportunity to optimize glycemic control with the goal of better outcomes, shorter hospital stays and lower overall cost of care.

\section{Summary}

The transition of care from the ED to the hospital medicine service represents a common step in the continuum of care but its impact may be underappreciated, especially in patients who present with DM and may have significant comorbidities. The clinical significance between DM and comorbid conditions is highlighted in the relationship between acute coronary syndrome and diabetes. Diabetes increases the risk of atherosclerosis in at least three ways and with this inextricable relationship between diabetes and cardiovascular disease in mind, identification of diabetes in patients who present with cardiovascular problems is essential to a smooth transition of care to the inpatient service. There is an opportunity to create a positive treatment continuum that begins in the ED and transitions to the inpatient service. This can be facilitated by the development of standard protocols to detect diabetes when the patient enters the ED and an ongoing collaborative relationship between the emergency physicians and the hospitalist or other hospital-based medical personnel. With these systems in place, the goal should be to provide optimal glycemic control for the patient from the time he or she arrives until discharge and thereafter.

\section{Compliance with Ethics Guidelines}

Conflict on Interest This paper was underwritten in part by a grant from Sanofi to the Hospital Quality Foundation to support the writing group's efforts, including travel. All authors report receiving consulting fees from Sanofi.

Human and Animal Rights and Informed Consent This article does not contain any studies with human or animal subjects performed by any of the authors.

\section{References}

Papers of particular interest, published recently, have been highlighted as:

- Of importance

•- Of major importance

1. • Giraldez RR, Clare RM, Lopes RD, et al. Prevalence and clinical outcomes of undiagnosed diabetes mellitus and prediabetes among patients with high-risk non-ST-segment elevation acute coronary syndrome. Am Heart J. 2013;165(918-925):e2. As the prevalence of diabetes increases, more patients may present with complications of the disease before the diabetes itself is diagnosed. ACS among prediabetics and undiagnosed diabetics is a prime example.

2. American Diabetes Association. Standards of Medical Care in Diabetes 2013. Hospital-based practitioners should be familiar with these standards.

3. O'Gara PT, Kushner FG, Ascheim DD, et al. ACCF/AHA guideline for the management of ST-elevation myocardial infarction. JACC. 2013;61(4):e78-140.

4. Donath MY, Shoelson SE. Type 2 diabetes as an inflammatory disease. Nat Rev Immunol. 2011;11:98-107.

5. Hansson GK. Inflammation, atherosclerosis, and coronary artery disease. N Engl J Med. 2005;352:1685-95.

6. Lumsden NG, Andrews KL, Bobadilla M, et al. Endothelial dysfunction in patients with type 2 diabetes post acute coronary syndrome. Diabetes Vasc Dis Res. 2013;10(4):368-74.

7. National Diabetes Fact Sheet 2011. CDC. Web Site. Available at: http://www.cdc.gov/diabetes/pubs/pdf/ndfs_2011.pdf. Accessed 21 Dec 2013.

8. - Baber U, Auguste U. Patients with chronic kidney disease/ diabetes mellitus: the high-risk profile in acute coronary syndrome. Curr Cardiol Rep. 2013;15:386-95. This article nicely explores the complex interrelationships among CKD, DM, and ACS.

9. Grundy SM. Pre-diabetes, metabolic syndrome, and cardiovascular risk. J Am Coll Cardiol. 2012;59:635-43.

10. Definition and Diagnosis of Diabetes Mellitus and Intermediate Hyperglycemia. Report of a WHO/IDF Consultation. WHO 2006. Web Site. Available at: http://www.who.int/diabetes/publications/ Definition\%20and\%20diagnosis\%20of\%20diabetes_new.pdf. Accessed 21 Dec 2013. 
11. Currie CJ, Poole CD, Peters JR, et al. Mortality and other important diabetes-related outcomes with insulin vs other antihyperglycemic therapies in type 2 diabetes. J Clin Endocrinol Metab. 2013;98:668-77.

12. Dawson AJ, Sathyapalan T, Atkin SL, et al. Biological variation of cardiovascular risk factors in patients with diabetes. Diabet Med. 2013;30(10):1172-80.

13. - Zaheer MS, Asjraf MU, Rabbani MU, et al. Prognostic importance of admission versus persistent glycemia in acute coronary syndrome. Diabetes Metab Syndr Clin Res Rev. 2013;7:42-7. This article highlights the benefits of tight glycemic control in ACS management.

14. Negendran J, Oudit GY, Bakal JA, et al. Are users of sulfonylureas at the time of an acute coronary syndrome at risk of poorer outcomes? Diabetes Obes Metab. 2013;15:1022-8.

15. Norhammar A, Schenck-Gustafsson K. Type 2 diabetes and cardiovascular disease in women. Diabetologia. 2013;56:1-9.

16. Niccoli G, Giubilato S, Di Vito L, et al. Severity of coronary atherosclerosis in patients with a first acute coronary event: a diabetes paradox. Eur Heart J. 2013;34:729-41.

17. Kannel WB, Hjortland M, Castelli WP. Role of diabetes in congestive heart failure: the Framingham study. AJC. 1974;34: 29-34.

18. Bertoni AG, Hundley WG, Massing MW, et al. Heart failure prevalence, incidence, and mortality in the elderly with diabetes. Diabetes Care. 2004;27(3):699-703.

19. Iribarren C, Karter AJ, Go AS, et al. Glycemic control and heart failure among adult patients with diabetes. Circulation. 2001; 103(22):2668-73.

20. Held C, Gerstein HC, Yusuf S, et al. Glucose levels predict hospitalization for congestive heart failure in patients at high cardiovascular risk. Circulation. 2007;115:1371-5.

21. Al-Aqeedi RF, Abdullatef WK, Dabdoob W, et al. The prevalence of metabolic syndrome components, individually and in combination, in male patients admitted with acute coronary syndrome, without previous diagnosis of diabetes mellitus. Libyan J Med. 2013;8:20185-91.

22. Beard JD. Chronic lower limb ischemia. West J Med. 2000;173: 60-3.

23. Clark N. Peripheral arterial disease in people with diabetes. Diabetes Care. 2003;26(12):3333-41.
24. Murabito JM, D'Agostino RB, Silbershatz H, et al. Intermittent claudication. A risk profile from The Framingham heart study. Circulation. 1997;96(1):44-9.

25. Williams LS, Rotich J, Qi R, et al. Effects of admission hyperglycemia on mortality and costs in acute ischemic stroke. Neurology. 2002;59:67-71.

26. Kim CS, Choi JS, Bae EH, et al. Association of metabolic syndrome and renal insufficiency with clinical outcome in acute myocardial infarction. Metabolism. 2013;62:669-76.

27. Griffin SJ, Borch-Johnsen K, Melanie J. Davies. Effect of early intensive multifactorial therapy on 5-year cardiovascular outcomes in individuals with type 2 diabetes detected by screening (ADDITION-Europe): a cluster-randomised trial. Lancet. 2011; 378:156-67.

28. Hanefeld M, Pfützner A, Forst T, et al. Double-blind, randomized, multicentre, and active comparator controlled investigation of the effect of Pioglitazone, Metformin, and the combination of both on cardiovascular risk in patients with type 2 diabetes receiving stable basal insulin therapy: the PIOCOMB study Cardiovascular. Diabetology. 2011;10:65-73.

29. Schramm TK, Gislason GH, Vaag A, et al. Mortality and cardiovascular risk associated with different insulin secretagogues compared with metformin in type 2 diabetes, with or without a previous myocardial infarction: a nationwide study. Eur Heart J. 2011;32(15):1900-8.

30. • Moghissi ES, Korytkowski MT, DiNardo M, et al. American Association of Clinical Endocrinologists and American Diabetes Association Consensus Statement on Inpatient Glycemic Control. Endocr Pract. 2009;15(4):1-17. This consensus statement makes the case for basal insulin therapy, especially in hospitalized diabetics.

31. Pittas J, Siegel RD, Lau J. Arch Intern Med. 2004;164:2005-11.

32. Cummings J, Mineo K, Levy R, et al. A review of the DIGAMI study: intensive insulin therapy during and after myocardial infarctions in diabetic patients. Diabetes Spectr. 1999;12(2):84-8.

33. Malmberg K, RydenL WH, et al. Intense metabolic control by means of insulin in patients with diabetes mellitus and acute myocardial infarction (DIGAMI 2): effects on mortality and morbidity. Eur Heart J. 2005;26:650-61.

34. Alverez-Sabin J, Molina CA, Arenillas J, et al. Effects of admission hyperglycemia on stroke outcome in reperfused tissue plasminogen activator-treated patients. Stroke. 2003;34:1235-40. 